\title{
Effects of the transcription factor Olig1 on the differentiation and remyelination of oligodendrocyte precursor cells after focal cerebral ischemia in rats
}

\author{
HONG ZHAO ${ }^{1}$, XIAO-YU GAO ${ }^{2}$, ZAN-HUA LIU ${ }^{3}$, JIAN-WEN LIN ${ }^{1}$, \\ SU-PING WANG ${ }^{1}$, DE-XIN WANG $^{4}$ and YONG-BO ZHANG ${ }^{4}$ \\ ${ }^{1}$ Department of Neurology, Dalian Municipal Central Hospital, Dalian, Liaoning 116033; \\ ${ }^{2}$ Department of Neurology, The Affiliated Yantai Yuhuangding Hospital of Qingdao University, Yantai, \\ Shandong 264000; ${ }^{3}$ Department of Neurology, Nanjing Gaochun People's Hospital, Nanjing, Jiangsu 211300; \\ ${ }^{4}$ Department of Neurology, Beijing Friendship Hospital, Capital Medical University, Beijing 100050, P.R. China
}

Received January 13, 2019; Accepted July 22, 2019

DOI: $10.3892 / \mathrm{mmr} .2019 .10713$

\begin{abstract}
The differentiation and maturation of oligodendrocyte precursor cells (OPCs) is important for remyelination in the central nervous system. Nevertheless, this process is often limited and incomplete in ischemic injury. Oligodendrocyte transcription factor 1 (Olig1) is important for the maturation of OPCs and the repair of demyelinated lesions. However, how Olig1 modulates the development of OPCs or the remyelination associated with ischemic injury remains unclear. The present study aimed to examine alterations in OPCs, and the expression of myelin and Olig1, at different time-points after focal cerebral ischemia using immunohistochemistry and western blot techniques to elucidate the role of Olig1 in the maturation of OPCs and remyelination. The present results showed that the expression of Olig1 significantly decreased at 1 day after middle cerebral artery occlusion (MCAO) and returned to normal levels from day 3 to 28 . Additionally, Olig1 was found to translocate into the nucleus following ischemia in the brain. The number of OPCs in the ischemic striatum significantly declined at days 1 and 3 following MCAO, and increased at days 7, 14 and 28 compared with the control. The expression of myelin basic protein, a marker of mature oligodendrocytes and myelin, gradually decreased from day 1 to 7 after ischemia and recovered at day 14 and 28; however, the levels were lower
\end{abstract}

Correspondence to: Dr Hong Zhao, Department of Neurology, Dalian Municipal Central Hospital, 826 Xi Nan Road, Dalian, Liaoning 116033, P.R. China

E-mail: zhaohong2003@126.com

Professor Yong-Bo Zhang, Department of Neurology, Beijing Friendship Hospital, Capital Medical University, 95 Yong An Road, Beijing 100050, P.R. China

E-mail: ybzhangcn@sina.com

Key words: oligodendrocyte precursor cells, oligodendrocyte, transcription factor Olig1, remyelination, differentiation, ischemia than those in the control group. The present results indicated that the restored normal level of Olig1 following ischemia may play an important role in the maturation of OPCs through its translocation into the nucleus, where it may promote the growth and development of myelin under pathological conditions. However, this endogenous recovery mechanism fails to fully repair the demyelinated lesion. The data of the present study may help clinicians understand the expression pattern of Olig1 and its potential role in endogenous remyelination after ischemia, which may have implications for the treatment of diseases that lead to demyelination.

\section{Introduction}

A number of previous studies have investigated the central white matter (1-3), which is predominantly composed of axons, which are surrounded by myelin. In the central nervous system (CNS), myelin is synthesized by oligodendrocytes, which wrap their membranes around axons to maintain saltatory conduction and neuronal support. Like neurons, oligodendrocytes are highly vulnerable to ischemic injury, which usually leads to loss and disruption of the myelin sheath $(4,5)$.

As a major source of new oligodendrocytes following ischemic stress, oligodendrocyte precursor cells (OPCs) are abundant throughout the gray and white matter of the adult brain (6-9). Previous studies have shown that OPCs are capable of rapidly dividing, migrating and eventually differentiating into myelin forming oligodendrocytes in response to local signals induced by stroke; this suggests that the adult brain possesses the capacity for endogenous remyelination by providing demyelinated areas with newly-formed mature oligodendrocytes (10-12). However, the capacity for spontaneous myelin repair is limited despite the abundance of OPCs, this may be due to the maturation arrest of OPCs $(13,14)$. Therefore, a better understanding of the molecular mechanisms that control the process of oligodendrocyte development is important to elucidate potential therapeutic interventions for demyelinating disorders. 
Olig1 and Olig2, two closely related basic helix-loop-helix transcription factors, have been identified and shown to play an important role in the differentiation of oligodendrocyte lineage cells from OPCs to mature oligodendrocytes (15-17). Previous gain and loss-of-function studies found that Olig2 is necessary for the initiation of oligodendrocytes, whereas Olig1 is suggested to contribute to the complete maturation of oligodendrocytes and to the repair of demyelinated lesions (18-21). Olig2 is present in the nucleus of oligodendrocyte at all developmental stages, while Olig1 is translocated from the nucleus to the cytoplasm in OPCs during normal myelinogenesis, and ultimately remains localized in the cytoplasm. Following demyelination, Olig1 is translocated back to the nucleus (22), suggesting a nuclear function of Olig1 that is important for remyelination. However, a detailed molecular mechanism of the translocation of Olig1 remains to be established. Previous studies revealed that the post-translation modification of proteins, particularly phosphorylation and acetylation, regulates the subcellular location of Olig1 $(23,24)$. Additionally, Tonchev et al (25) reported an increase in the number of Olig1-immunopositive (Olig1+) cells in the subventricular zone following ischemia. It is possible that both the intracellular redistribution of Olig1 and regulation of its expression levels may be required for the repair process, however, these mechanisms have not been well studied in the middle cerebral artery occlusion (MCAO) model. Exploring the temporal and spatial expression and distribution of Olig1 will increase the understanding of the role of Olig1 in oligodendrogenesis and facilitate the development of novel therapeutic strategies for remyelination.

Ischemia acutely induces mature oligodendrocyte damage, leading to the diffuse loss of myelin and axons, which is associated with severe neurologic deficits (9). OPCs contribute to myelination in the CNS and have received much attention regarding their role in ischemic-associated injuries (10). During recovery from ischemia, a significant increase in the number of OPCs is observed, and some of them become mature myelinating oligodendrocytes, in peri-infarct gray and white matter where sprouting axons are present (12). Transcriptional factor Olig1 was detected in oligodendrocytes and OPCs of the CNS and regulated oligodendrocyte lineage progression (18). Although previous studies have demonstrated that the role of Olig1 in the development of OPCs $(17,22)$, the relationship between Olig1 and OPCs development, and myelination, remains largely unknown. In the present study, the expression patterns of Olig1, along with the alteration of OPCs and myelin, were systematically observed in the brain following ischemia insult (from 1 to 28 days) using immunohistochemistry and western blot analysis. The effects of Olig1 on the maturation of OPCs and the subsequent remyelination in a rat focal ischemic model were discussed in detail. The present study provided an insight for the restorative treatment of ischemia or other demyelination disease that requires the manipulation of endogenous oligodendrogenesis in order to enhance spontaneous brain repair.

\section{Materials and methods}

Experimental animals. A total of 72 adult male Sprague-Dawley rats at age 6-8 weeks weighing 260-280 g were used in the present study and randomly assigned to the ischemic group or the control group. The rats were purchased from the Department of Experimental Animal Sciences, Dalian Medical University, and housed under standard laboratory conditions (temperature, $20-25^{\circ} \mathrm{C}$; relative humidity, $50-70 \%$ ) for 7 days before experimentation. The animals had free access to food and water during the experiment, and were maintained in natural day/night cycles. Efforts were made to minimize the discomfort of the animals. All protocols were approved by Animal Welfare Committee of Dalian Medical University and followed the guidelines for Animal Care and Use adapted from the National Institutes of Health.

Surgery. The rats were anesthetized with $80 \mathrm{mg} 10 \%$ chloral hydrate $(300 \mathrm{mg} / \mathrm{kg})$ by intraperitoneal injection, no signs of peritonitis were observed in the animals. The middle cerebral artery (MCA) was occluded as described by Longa et al (26). After midline incision of the neck skin, the right external carotid artery (ECA) was carefully isolated from the surrounding nerves and fascia. Briefly, a nylon monofilament suture of $0.26 \mathrm{~mm}$ diameter (with one end rounded by heat and a diameter of $0.34 \mathrm{~mm}$ ) was inserted into the ECA and advanced into the internal carotid artery to obstruct the origin of the right MCA. The suture was inserted $18-20 \mathrm{~mm}$ from the bifurcation of the common carotid artery. The rectal temperature of the animals was maintained at $37 \pm 0.5^{\circ} \mathrm{C}$ using a heat lamp during the operation. After awakening, neurological deficits were evaluated according to the method of Bederson et al (27). Rats without left forelimb paresis or circling towards the left side were regarded as unsuccessful models and were excluded from further study. The rats were anesthetized with ether $90 \mathrm{~min}$ after MCAO and the occluding filament was withdrawn. The rats in the control group were subjected to isolation of their right carotid arteries only. At 1, 3, 7, 14 and 28 days after MCAO, the rats were anesthetized with $80 \mathrm{mg} 10 \%$ chloral hydrate $(300 \mathrm{mg} / \mathrm{kg})$ followed by decapitation or by perfusion (12 rats at each time-point and 12 total control rats).

Immunohistochemistry staining. Rats were perfused with normal saline followed by $4 \%$ paraformaldehyde (PFA) in $0.1 \mathrm{M}$ phosphate buffer (PB, $\mathrm{pH} 7.4)$ for $20 \mathrm{~min}$ at $25^{\circ} \mathrm{C}$. The brains were removed, post-fixed in $4 \%$ PFA for $24-48 \mathrm{~h}\left(4^{\circ} \mathrm{C}\right)$ and then transferred to PB with $30 \%$ sucrose $\left(4^{\circ} \mathrm{C}\right)$. Several days later, the brains were frozen in solid carbon dioxide and then cut in $20-\mu$ m-thick serial sections using a cryostat (Cryocut1800; Leica Microsystems, Inc.). The sections were mounted on gelatin/chrome alum-coated glass slides.

The slides were processed for Olig1, NG2 or myelin basic protein (MBP) using the avidin-biotin technique (Elite kit; Vector Laboratories, Inc.), as described by Tu et al (28). The sections were blocked with $2 \%$ goat serum (OriGene Technologies, Inc.) for $30 \mathrm{~min}$ at $37^{\circ} \mathrm{C}$ and then incubated overnight at $4^{\circ} \mathrm{C}$ in a humid chamber with the following antibodies: Olig1 (1:400; MAB5540, Chemicon International; Thermo Fisher Scientific, Inc.), NG2 (1:400; AB5320, Chemicon International; Thermo Fisher Scientific, Inc.) or MBP (1:200; BA0094; Wuhan Boster Biological Technology, Ltd.). After washing in $0.01 \mathrm{M}$ PBS three times, sections were incubated with goat anti-mouse or goat anti-rabbit secondary antibodies conjugated to HRP (1:100, SP9001 or SP9002, 
OriGene Technologies, Inc.) for $30 \mathrm{~min}$ at $37^{\circ} \mathrm{Cand}$ were finally processed with diaminobenzidine $\left(1 \mathrm{mg} / \mathrm{ml} ; 0.001 \% \mathrm{H}_{2} \mathrm{O}_{2}\right)$ for $2 \min$ at $25^{\circ} \mathrm{C}$.

Images were captured with a charge-coupled device (CCD) spot light camera under x100, x200 and x400 magnification with light microscopy (BX51; Olympus Corporation). MetaMorph software (v7.8; Molecular Devices, LLC) was used for the quantification of cell number and the average intensity in the region of the ischemic striatum.

For the analysis of Olig1 expression in normal rats, 3 sections in different brain areas (the cerebral cortex, corpus callosum, and the striatum and hippocampus) were selected and 5 fields of each section at the same magnification were randomly chosen to count Olig1 positive cells. The total number of $\mathrm{Olig} 1^{+}$cells was obtained from three sections of each brain area and then averaged for six rats.

An area of 1,400x1,200 $\mu \mathrm{m}$ in the ischemic striatum was defined for counting Olig1 or NG2 positive cells at different time-points after MCAO. The slides were visualized by light microscope under x100 magnification. Counting of the number of Olig1 and NG2 cells was carried out blindly. In total, 4 slides from each rat brain were obtained from $20 \mu \mathrm{m}$ thick coronal sections between $1.4 \mathrm{~mm}$ anterior and $0.4 \mathrm{~mm}$ posterior to the bregma. All counts were pooled and results were expressed as the average number of positive cells per rat. In total, 6 rats were studied per group.

The average intensity of MBP-immunoreactivity in the striatum of the ischemic side was calculated to identify the extent of remyelination. The average intensity was defined as the difference between the average gray value (mean density) within the ischemic striatum and its background (29).

Western blot analysis. At 1, 3, 7, 14 and 28 days after reperfusion, the animals were sacrificed and the levels of Olig1 and MBP were determined. Western blot analysis was performed as described by Jiang et al (30). Briefly, the bilateral striatum of the brain were removed, quickly frozen in liquid nitrogen and stored at $-80^{\circ} \mathrm{C}$ for later use. Frozen tissues were homogenized in homogenization buffer $(50 \mathrm{mmol} / \mathrm{liter}$ Trisxbase, $2 \mathrm{mmol} / \mathrm{liter}$ EDTA, $40 \mathrm{mmol} / \mathrm{liter} \mathrm{NaF}, 1 \mathrm{mmol} / \mathrm{liter}$ phenylmethylsulfonyl fluoride). Protein concentrations were determined using the bicinchoninic acid method. Samples containing $60 \mathrm{mg}$ of protein were separated by $15 \%$ SDS-PAGE and transferred to PVDF membranes. The blots were blocked with $5 \%$ non-fat milk for $1 \mathrm{~h}$ at $25^{\circ} \mathrm{C}$ and incubated with mouse monoclonal anti-Olig1 antibody (1:4,000; MAB5540; Chemicon International; Thermo Fisher Scientific, Inc) or rabbit polyclonal anti-MBP antibody (1:1,000; BA0094, Wuhan Boster Biological Technology, Ltd.) overnight at $4^{\circ} \mathrm{C}$. $\beta$-actin (1:8,000; sc-130065; Santa Cruz Biotechnology, Inc.) served as an internal control. After washing three times with TBST, membranes were incubated for $1 \mathrm{~h}$ at room temperature with goat anti-mouse or goat anti-rabbit IgG-HRP (1:1,000; sc-2005 or sc-2004; Santa Cruz Biotechnology, Inc.). Protein bands were visualized using enhanced chemiluminescence reagents (Santa Cruz Biotechnology, Inc.) and the membranes were exposed to autoradiographic film for 1-5 min.

For the quantification of the western blotting signals, the blots were scanned with ScanWizard 5.0 (Microtek International, Inc.) and the density of the bands were measured with the TotalLab version 1.0 software (TotalLab Ltd.). Values for Olig1 or MBP were normalized to the intensity of $\beta$-actin.

Statistical analysis. The experiments were repeated three times. Data are expressed as the mean \pm SEM. Statistical analysis was performed using GraphPad Prism (version 5.0; GraphPad Software, Inc.). Differences between the control and various time-points were determined using one-way ANOVA followed by Dunnett's post-hoc test. $\mathrm{P}<0.05$ was considered to indicate a statistically significant difference.

\section{Results}

Expression pattern of Oligl protein in control and ischemic rats. Fig. 1 shows the expression pattern of Olig1 protein in control and ischemic brain sections with immunohistochemical staining. Olig1-immunoreactive cells were widely distributed throughout the gray and white matter of the control adult brain, including the cerebral cortex, corpus callosum, the striatum and hippocampus (Fig. 1A and B). Olig1-immunoreactivity in the control rats was restricted predominantly to the cytoplasm and rarely seen in the nuclei of oligodendroglial-like cells (characterized by a small cell body, sparse cytoplasm and few processes; Fig. 1C and D). After focal ischemia, however, the nuclear localization of Olig1 significantly increased in the area surrounding lesions (Fig. 1E and F). This indicated that the Olig1 protein was recruited to the nucleus following MCAO.

Time course of Oligl-immunoreactivity after MCAO. Fig. 2A is a representative example of the immunostaining for Olig1-immunoreactivity in the right striatum on the sham-operation day (control) and on days 1, 3, 7, 14 and 28 after MCAO. The statistical results are shown in Fig. 2B. It was found that the number of Olig1-immunoreactive cells were reduced at days 1 and 3 after MCAO, with lowest numbers observed at day 1 . The number of Olig1-immunoreactive cells increased at day 7 and remained at this level for the next 4 weeks. The ischemic side of MCAO rats exhibited extensive nuclear Olig1 staining, as observed by light microscopy, from day 1 to 28 following the operation. By contrast, the contralateral side showed no evidence of Olig1 redistribution from the cytoplasm to the nucleus in oligodendrocyte (data not shown), which indicated that the presence of nuclear Olig1 is highly correlated with the active lesions.

Western blotting was performed to analyze Olig1 expression. Results in the ischemic striatum at different time-points (up to 28 days) are shown in Fig. 3A and B. Consistent with the findings of the immunohistochemistry, Olig1 expression significantly decreased at 1 day after MCAO, returned to a near control level at day 3 and maintained this level throughout the remaining period of the experiment. However, there was no difference in the expression of Olig1 between control and MCAO rats in the contralateral striatum at any time-point (Data not shown).

Morphological changes of OPCs in the ischemic striatum of the rats. Fig. 4A shows the population of OPCs in the ischemic area, which was stained using an NG2 antibody. The number of OPCs was uniformly distributed in the white and gray matter of the control brain. These cells had small bodies, round or 


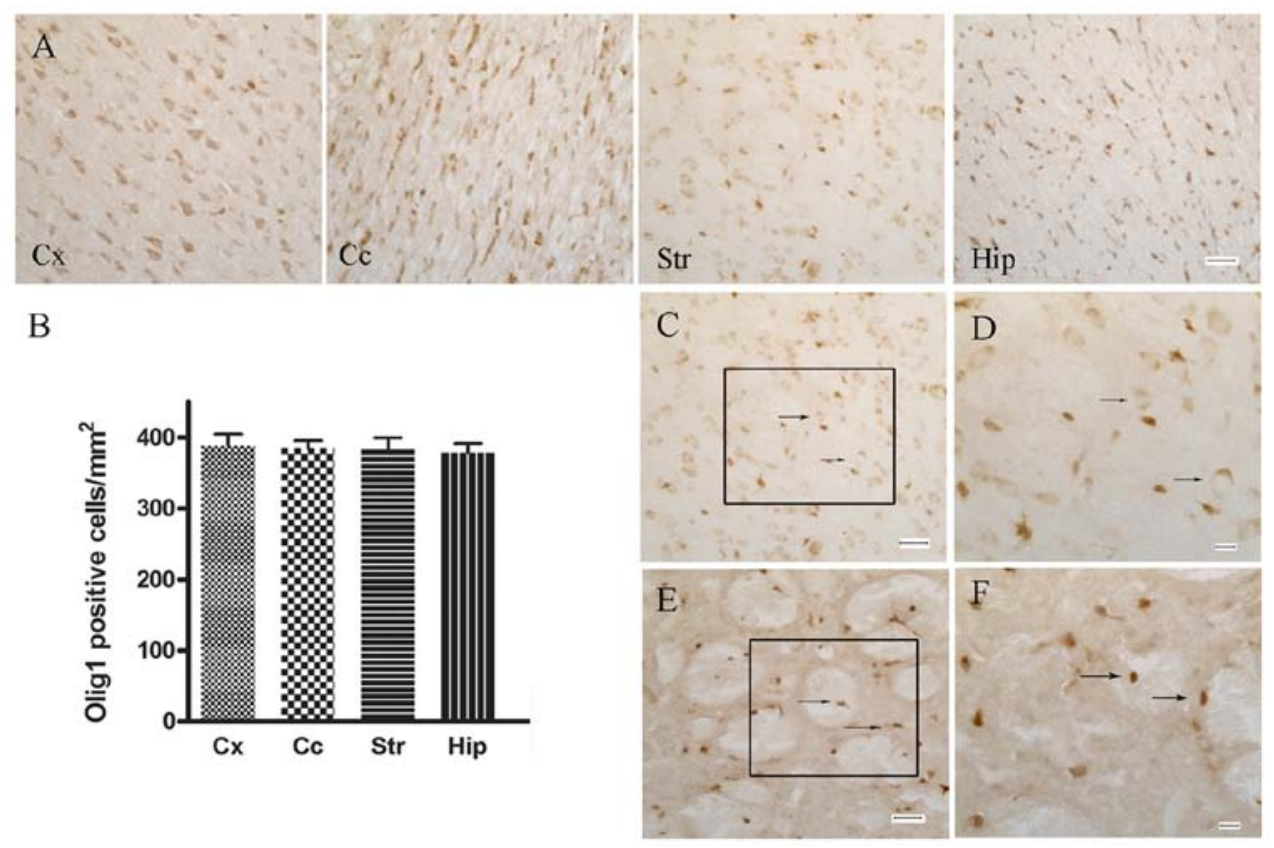

Figure 1. Immunohistochemical staining of Olig1 in control and MCAO rats. (A) Distribution of Olig1 positive cells in different regions of the brain. Scale bar, $30 \mu \mathrm{m}$. (B) Statistical analysis of (A). (C) In normal rats, Olig1 was predominantly located in the cytoplasm and rarely in the nuclei of oligodendrocytes. Arrows indicate the cytoplasmic localization of Olig1. Scale bar, $30 \mu \mathrm{m}$. (D) Higher magnification of black box in (C). Scale bar, $10 \mu \mathrm{m}$. (E) MCAO rats exhibited extensive nuclear localization of Olig1. Arrows indicate the nuclear localization of Olig1. Scale bar, $30 \mu \mathrm{m}$. (F) Higher magnification of black box in (E). Scale bar, $10 \mu \mathrm{m}$. Olig1, oligodendrocyte transcription factor 1; MCAO, middle cerebral artery occlusion; Cx, cortex; Cc, corpus callosum; St, striatum; hip, hippocampus.

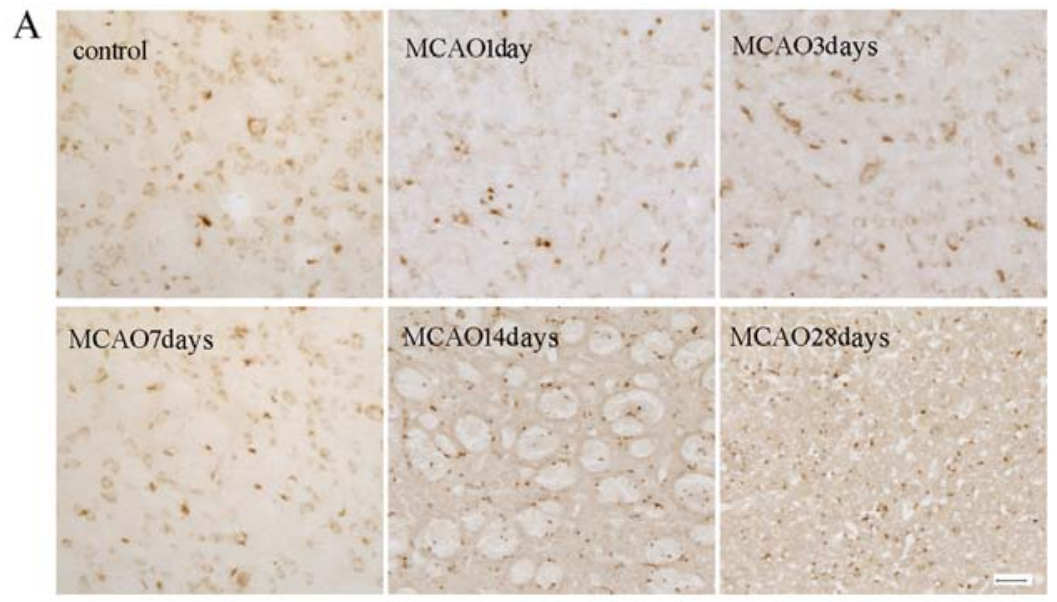

B

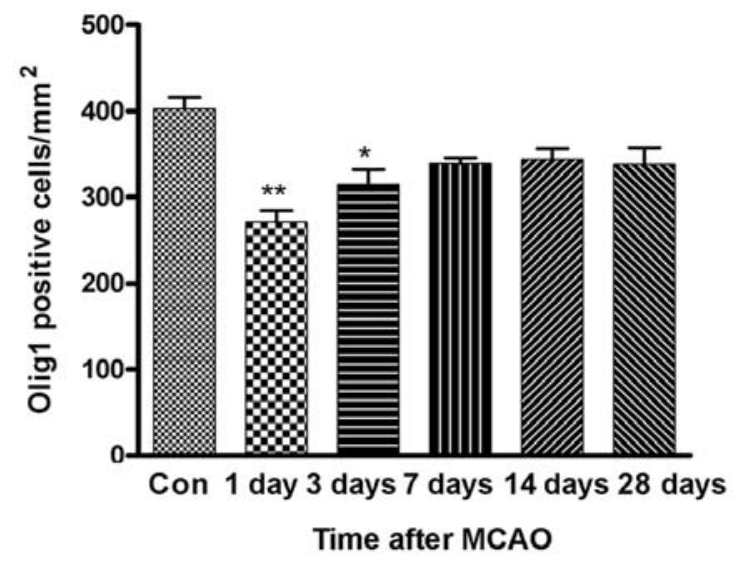

Figure 2. Time course of Olig1 immunostaining in the ischemic striatum following MCAO. (A) Olig1 immunostaining at days 1,3, 7, 14 and 28. The number of Olig1 positive cells decreased 1 day after MCAO and then returned to control levels at day 3 . This level was maintained until day 28 . Nuclear Olig1 was significantly increased in the striatum of the ischemic side after MCAO. Scale bar, $50 \mu \mathrm{m}$. (B) Quantification of the number of Olig1 positive cells. $\mathrm{n}=6$. ${ }^{*} \mathrm{P}<0.05,{ }^{* *} \mathrm{P}<0.01$ vs. Con. Olig1, oligodendrocyte transcription factor 1 ; MCAO, middle cerebral artery occlusion; Con, control. 
A

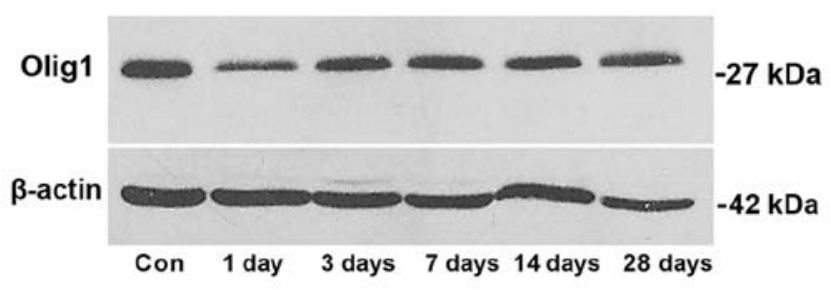

B

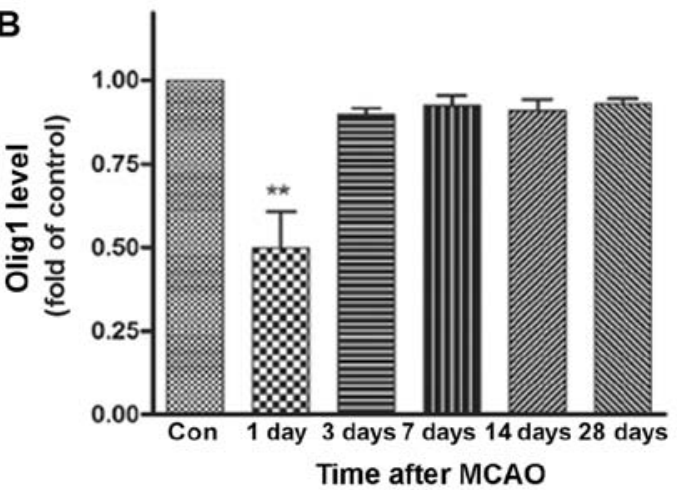

Figure 3. Western blot analysis of Olig1 in the striatum after MCAO. (A) Olig1 bands in the Con group and at different time points (1, 3,7 , 14 and 28 days following focal cerebral ischemia) in the ischemic striatum. The level of Olig1 protein decreased 1 day after MCAO and then returned to near control levels from day 3 to 28 . $\beta$-actin was used as a loading control. (B) Quantification of results in (A). $\mathrm{n}=6$. $^{* *} \mathrm{P}<0.01$ vs. Con. Olig1, oligodendrocyte transcription factor 1 ; $\mathrm{MCAO}$, middle cerebral artery occlusion; Con, control.

oval, with multiple and highly branched processes extending in all directions. In the brains sampled 7 days after MCAO, the OPCs exhibited swollen cell bodies, with very heavy staining, and many short and hypertrophied processes, indicating OPCs were activated and entered mitosis. The number of NG2-positive cells was quantified in the ischemic region at each of the time-points (Fig. 4B). The number of OPCs in the ischemic striatum significantly decreased at days 1 and 3 , and recovered at day 7 . The number of OPCs reached a peak at day 14, and this level was maintained until day 28 after MCAO.

Changes in the expression of MBP in brain tissues. To investigate the extent of demyelination in the white matter of MCAO rats, the expression of MBP, a major constituent of CNS myelin, was studied using immunohistochemistry and western blotting. Representative images from the time course of MBP staining in ischemic striatum is shown in Fig. 5A. Many myelinated fibers, which were detected with anti-MBP antibody, were clearly visible in the control cerebral striatum and each fiber tract could be easily followed. By contrast, these fibers became obscure and spaces between fiber tracts appeared during reperfusion following MCAO. The ratio of MBP optical densities on the ischemic side was also analyzed (Fig. 5B). The average intensity of MBP staining in the ischemic group was decreased gradually from day 1 to 7 , and marginally recovered at days 14 and 28. However, the difference between days 14 and 28 was not statistically significant $(\mathrm{P}>0.05)$.

Results of the western blotting and band density analysis are shown in Fig. 6A and B. MBP levels in the affected striatal tissues were measured at days 1, 3, 7, 14 and 28 after MCAO. Compared with the control, the average level of MBP progressively decreased from day 1 to 7 , reaching a minimum at day 7 , then recovered at days 14 and 28. Despite the increased expression of MBP at days 14 and 28, the MBP expression level remained lower than in the control group. These data suggested that myelin loss occurred at an early time-point (1 day) and advanced during ischemia.

\section{Discussion}

OPCs are defined as glial cells in the CNS and constitute a stable population of quiescent cells that divide infrequently (6).

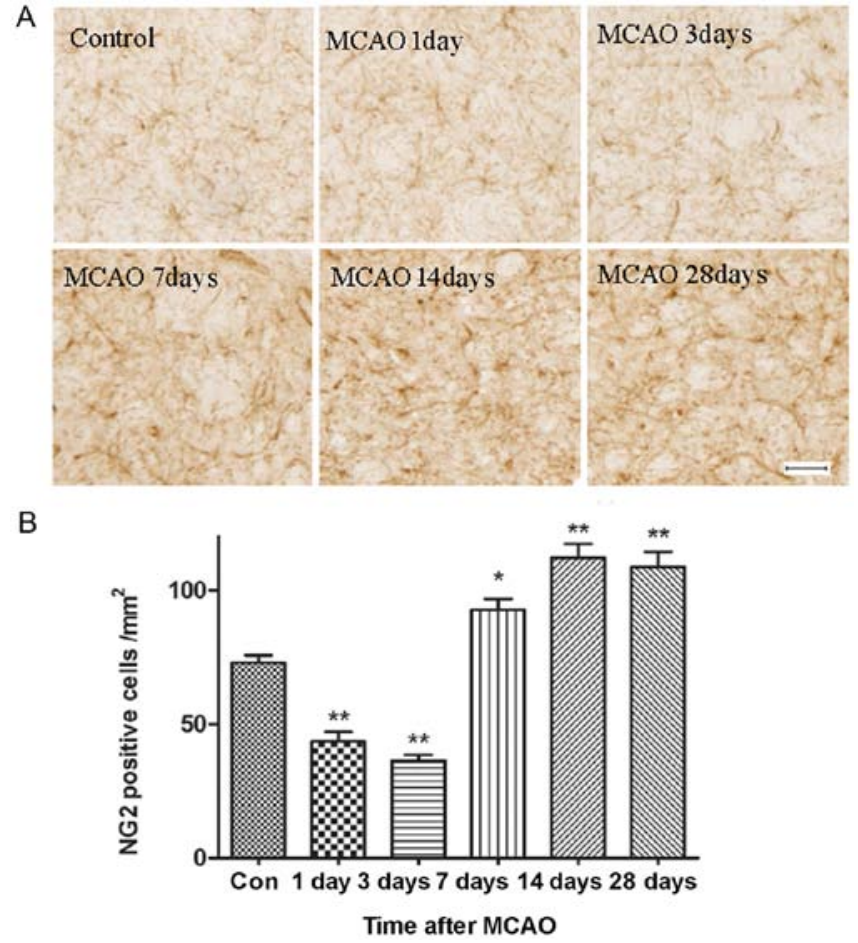

Figure 4. Immunostaining of OPCs in the ischemic area. (A) Representative images of sections stained with anti-NG2, a marker of OPCs, at day 1, 3, 7, 14 and 28 after reperfusion. Scale bar, $50 \mu \mathrm{m}$. There was a significant increase in the number of OPCs and this was accompanied by morphological changes in these cells from day 7 to 28 after reperfusion. (B) Statistical analysis of the number of NG2 positive cells. $\mathrm{n}=6 .{ }^{*} \mathrm{P}<0.05,{ }^{* *} \mathrm{P}<0.01$ vs. Con. MCAO, middle cerebral artery occlusion; Con, control; OPCs, oligodendrocyte precursor cells; NG2, chondroitin sulfate proteoglycan 4.

Remyelination is a complicated and elaborate process, which is predominantly mediated by endogenous OPCs (10). In response to injury signals at sites of demyelination, OPCs in the vicinity are activated. OPCs then proliferate and migrate into the injured area and differentiate into oligodendrocytes, which form new myelin sheaths wrapping the demyelinated axons (11,31-33). This indicates that the adult brain possesses the capacity to regenerate myelin by supplying mature oligodendrocytes that originate from activated OPCs. Accordingly, the OPCs in the adult brain are hypothesized to have the potential 


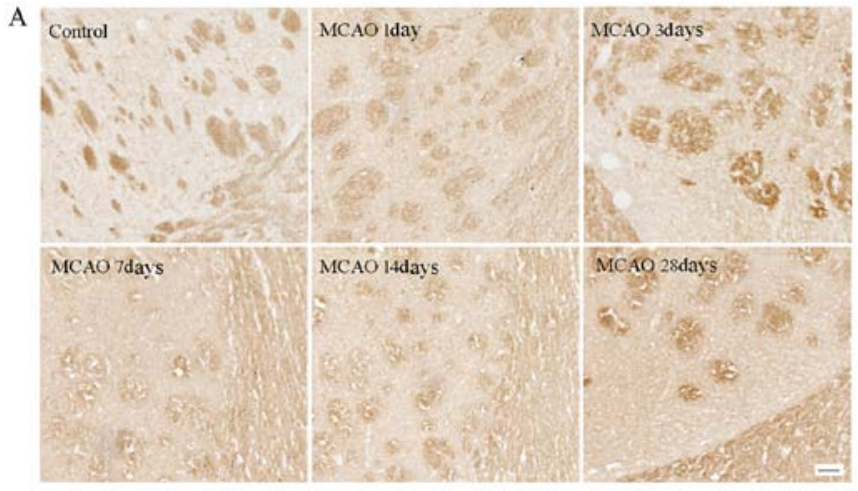

B

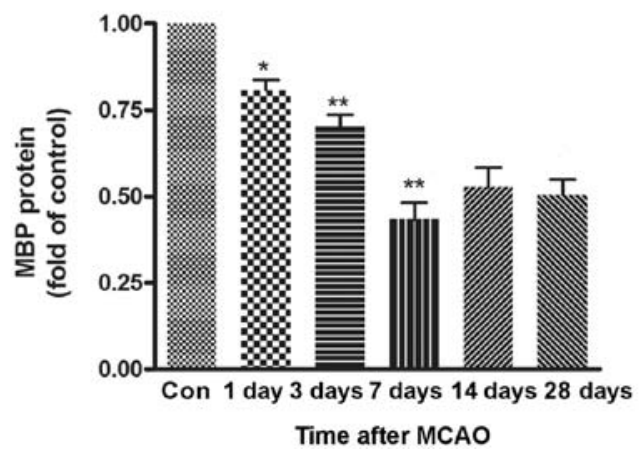

Figure 5. Measurement of MBP expression in the ischemic brain tissues using immunohistochemistry. (A) Representative images of sections stained with anti-MBP, a marker for myelin, after MCAO. Scale bar, $50 \mu \mathrm{m}$. (B) Quantification of the average intensity as shown in (A). The intensity of MBP decreased from day 3 to 28 after MCAO. $\mathrm{n}=6 .{ }^{*} \mathrm{P}<0.05,{ }^{* *} \mathrm{P}<0.01$ vs. Con. MBP, myelin basic protein; MCAO, middle cerebral artery occlusion; Con, control.

to repair damaged brain tissue. Tanaka et al (12) showed that the upregulation in the number of OPCs may contribute to an almost complete recovery of the myelin in the peri-infarct area after ischemic insult. However, previous studies demonstrated that remyelination is limited and incomplete, although OPCs are present $(13,14)$. These inconsistent results indicate that it is necessary to further explore the contribution of OPCs to myelin repair following ischemia, with the aim of identifying potential targets for clinical treatment.

In the present study, changes in OPCs and myelin were observed systematically from day 1 to 28 after MCAO. The findings of the present study revealed that OPCs stained with an anti-NG2 antibody were abundant and homogenously distributed in the white and gray matter of adult rat brains. These cells persist quiescently and have small bodies with multiple, and highly branched, processes under normal conditions. Consistent with previous studies $(12,34)$, we found that OPCs exhibited swollen and hypertrophied cell bodies with many radiating thick processes when the brain was subjected to ischemia insult, indicating OPCs were activated and entered into the mitotic phase in response to damage. This transition from a quiescent to an activated state enabled OPCs to undergo differentiation, which is important to the success of remyelination. In addition, these morphological changes were accompanied by a significant increase in the number of OPCs. As shown in the present study, the number of OPCs reduced significantly between day 1 and 3 after reperfusion, and then increased until day 28. A significant decrease in the population of OPCs at the early stage following reperfusion suggested that OPCs may be as vulnerable as neurons to ischemia. The ischemic striatum demonstrated a gradual increase in the number of OPCs, possibly because the activated OPCs migrated into the injury sites from the outer pre-infarct area to substitute the loss of these cells. Although a number of OPCs were recruited into the ischemic striatum, whether they could differentiate terminally into mature oligodendrocytes and form new myelin remains to be determined. MBP was used as a specific marker of mature oligodendrocytes and myelin sheath (35). It was found that MBP expression decreased from day 1 to 7 following focal cerebral ischemia, and marginally recovered at days 14 and 28 (Figs. 5 and 6), which indicated a loss of myelin at day 1 and a continuous advancement of demyelination until day28. Despite the late increase, the MBP expression level remained lower than that of the control group, suggesting that the injured myelin can only be partially repaired. The limited remyelination indicated that OPCs maturation may be inhibited under ischemic condition. This is supported by the fact that Olig1 is transported into the cytoplasm following oligodendrogenesis $(22,36,37)$; however, in the present study, most of the Olig1 protein remained in nucleus as late as 28 days after ischemia, suggesting that OPCs maturation was hindered. The molecular mechanisms regulating OPC differentiation and maturation under pathological conditions require further investigation.

Olig1 is important for oligodendrocyte differentiation from OPCs to mature oligodendrocytes and the repair of demyelinated lesions $(36,38)$. The expression pattern of Olig1 and its effects on remyelination were investigated. It was observed that Olig1 was located predominantly in cells with an oligodendroglial morphology (small process-bearing cells) and widely distributed homogeneously throughout the white and gray matter of the adult brain. This is consistent with other reports $(16,39)$. That the expression of Olig1 persists throughout the life of an oligodendrocyte suggests a fundamental role for this gene in oligodendrocyte maturation and/or survival (40). The fact that oligodendrocytes fail to develop in Olig1 knockout mice supports this viewpoint $(19,20)$. Furthermore, the present study demonstrated that in the normal brain, the Olig1 protein was cytoplasmic. According to the subcellular localization of Olig1 at different developmental stages, it was predicted that the small proportion of cells containing nuclear Olig1 is likely to be the quiescent undifferentiated progenitors, which are abundant and comprise $5-8 \%$ the glial cell population in the normal brain (41). The function of these nuclear Olig1+ cells in the undamaged CNS is unclear, however, it may involve spontaneous remyelination, as suggested in the human CNS during aging $(42,43)$. In the present study, when the brain underwent ischemia, Olig1 displayed extensive nuclear localization in the affected striatum, consistent with a previous report (22). However, the meaning of the dynamic redistribution of Olig1 is poorly understood. It was shown that the phosphorylation of Olig1 regulated its nuclear-cytoplasmic shuttling during oligodendrocyte development. Olig1 localized into the nucleus to facilitate myelin gene expression, then Olig1 changed the phosphorylation state and translocated into the cytoplasm to enhance membrane expansion and differentiate into mature oligodendrocytes (24). These findings imply that the cytoplasmic relocation of Olig1 may contribute to the process by which OPCs mature into oligodendrocytes. 
A

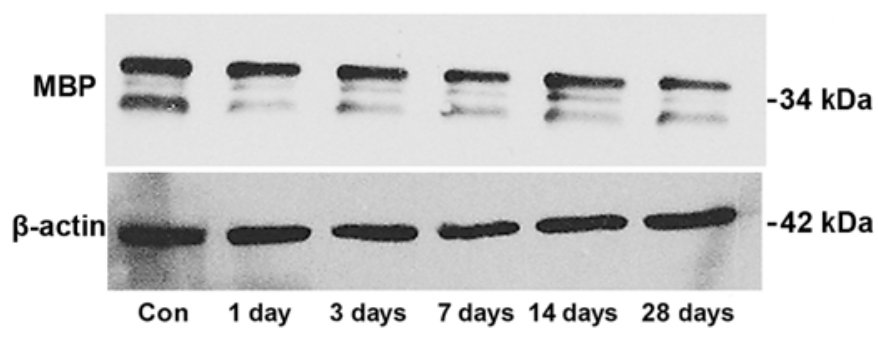

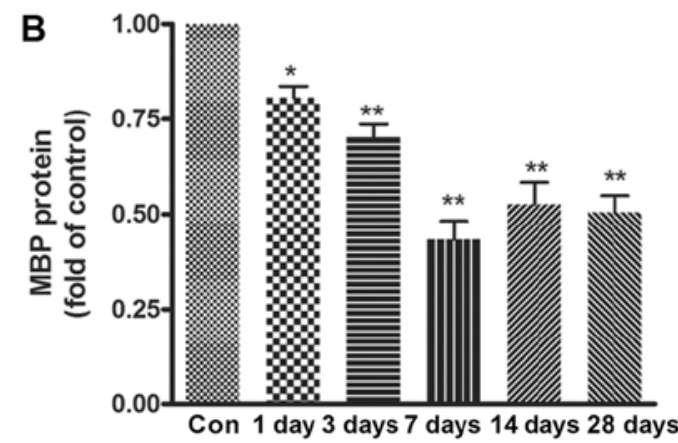

Figure 6. Western blot analysis of MBP in the ischemic striatum of MCAO rats. (A) MBP expression in the Con group and at different time points (1, $3,7,14$ and 28 days) after MCAO. (B) Quantification of the results shown in (A). The expression of MBP decreased from day 1 to 7 , with the lowest expression at day 7. MBP expression increased at days 14 and 28, however, it remained lower than the control group. $\mathrm{n}=6$. $^{*} \mathrm{P}<0.05$, ${ }^{* *} \mathrm{P}<0.01$ vs. Con. MBP, myelin basic protein; MCAO, middle cerebral artery occlusion; Con, control.

However, the detailed molecular mechanism of this process requires further investigation.

For the first time in the present study, to the best of our knowledge, a systematic and long-term observation of Olig1 expression after $\mathrm{MCAO}$ was performed. Using immunostaining it was found that the number of Olig $1^{+}$cells decreased from day 1 to 3 after MCAO, and recovered to a near normal level until day 28. Western blotting analysis supported the results of the immunostaining. The mechanism mediating the rapid reduction in the number of Olig $1^{+}$cells at day 1 after ischemia is likely to be complex. As Olig1 is predominantly expressed by OPCs and myelinating oligodendrocytes $(16,39)$, the death of these cells within the lesion area may explain the reduced number of Olig1 ${ }^{+}$cells, although it cannot be excluded that the transcriptional downregulation of Olig1 may also contribute to this. The increased number of Olig $1^{+}$cells observed from day 7 to day 28 after ischemia may be due to the recruitment and/or proliferation of Olig1 ${ }^{+}$OPCs at the injury sites, which may partially compensate for Olig $1^{+}$cells death.

The transcription factor Olig1 is an important regulator of OPCs and can promote the development of immature OPCs to mature oligodendrocytes, and eventually contribute to the migration of mature of oligodendrocytes to newly formed myelin (44). The present study showed a sharp decline in the expression of Olig1 and MBP at 1 day following the ischemia insult. Subsequently, the expression of Olig1 was restored to near normal levels from day 3 to 28, while MBP expression decreased from day 3 to 7 and recovered marginally until day 28 .

Despite the partial recovery at the late stage of ischemia, the expression of MBP expression failed to reach the levels observed in the control group. The expression of MBP increased at the late compared to the early stage, which is a pattern similar to that of Olig1; this suggested that Olig1 has a promoting role in the repair and regeneration of myelin. These findings revealed that the downregulation of Olig1 in hypoxic-ischemic brain tissue led to a decreased expression of some proteins, such as MBP, that are required to form myelin, and thus delayed the synthesis of myelin. However, despite the repair effect of Olig1, myelin was not fully repaired to a normal level. Our previous study revealed that the upregulation of Olig1 may enhance the differentiation of OPCs into mature oligodendrocytes and accelerate the kinetics of myelination after focal cerebral ischemia (45), indicating that the normal level of Olig1 is not sufficient for myelinogenesis following ischemia. The effect of the upregulation of Olig1 on the repair process following ischemia requires further investigation.

Olig1 is an important regulator of myelin specific genes, however, the changes of Olig1 expression did not parallel those of MBP, leading to the speculation that Olig1 regulates the expression of MBP along with other transcription factors. The process governing remyelination is complex and orchestrated by a network of transcriptional regulators; Olig1 as a single transcription factor is not sufficient (46). It has been reported that increased expression of the homeobox protein $\mathrm{Nkx} 2.2$ and Olig2 had an important role in the differentiation of OPCs into remyelinating oligodendrocytes (47). An in vitro experiment showed that oligodendrocyte differentiation initiated by Olig1 gene transfection did not lead to full maturation, which highlighted requirement for Nkx2.2 (48). Moreover, other transcription factors, such as Sox9, Sox10, Nkx2.2 and zinc finger protein 488 , may be involved in regulation (49-51). It was found that $\mathrm{Nkx} 2.2^{+} / \mathrm{Olig} 2^{+} \mathrm{OPCs}$ failed to remyelinate in areas depleted of astrocytes, which suggested astrocytes may produce a wide range of signaling molecules to support differentiation $(52,53)$. However, glial scars composed of astrocytes may prevent remyelinating cells from gaining access to demyelinated axons, so it is not yet clear whether astrocytes promote or hinder the process $(54,55)$. In addition, the existence of myelin inhibitory factor and the delayed clearance of myelin debris surrounding demyelinated axons by macrophages also have inhibitory effects on remyelination (56). Processes governing remyelination rely not only on the intrinsic determinants expressed in a specific spatial and temporal sequence, but also on extracellular signals.

In conclusion, the present study investigated the changes of Olig1 expression in MCAO rats and further explored the effects of Olig1 on OPC maturation, and remyelination, using immunostaining and western blotting. The present study has shown for the first time, to the best of our knowledge, that Olig1 is markedly decreased during the initial $24 \mathrm{~h}$ after demyelination injury, and is subsequently returned to near control levels, which are maintained up to day 28. Olig1 translocated from cytoplasm to nucleus at the ischemia site. The number of OPCs increased from day 7 to 28 after ischemic insult, while the expression of MBP decreased from day 1 to 7 and marginally increased at days 14 and 28. Despite the increase at 
the late time-points following MCAO, the final levels of these proteins remained lower than the corresponding levels in the control group; this suggested that Olig1 has a limited ability to promote the repair of myelin. In the present study it was found that Olig1 can promote myelination by stimulating nuclear transcription, but this effect is insufficient for the complete repair of the damaged myelin. Additional studies are required to determine whether interventions based on Olig1 expression or stimulating nuclear Olig1 relocation into the cytoplasm to promote endogenous remyelination would be efficient to prevent and/or treat ischemic-related disease.

\section{Acknowledgements}

The authors would like to thank Professor Jie Li (University of Cincinnati) for helpful corrections to the manuscript. The authors would also like to thank Dr You Wan (The Neuroscience Research Institute, Peking University) for technical assistance.

\section{Funding}

The current study was supported by grants from The National Natural Science Foundation of China (grant nos. 30570626, 81371355 and 81671191) and The Beijing Natural Science Foundation (grant no. 7082028).

\section{Availability of data and materials}

The datasets used and/or analyzed during the current study are available from the corresponding author on reasonable request.

\section{Authors' contributions}

HZ and YBZ conceived and designed the study. XYG and ZHL performed the experiments. JWL, SPW and DXW analyzed the data and drafted the manuscript. All authors have read and approved the final manuscript.

\section{Ethics approval and consent to participate}

The animal-related experiments were approved by Animal Welfare Committee of Dalian Medical University and followed the guidelines for Animal Care and Use adapted from NIH, USA.

\section{Patients consent for publication}

Not applicable.

\section{Competing interests}

The authors declare that they have no competing interests.

\section{References}

1. Marin MA and Carmichael ST: Stroke in CNS white matter: Models and mechanisms: Neurosci Lett 684: 193-199, 2018.

2. Goldberg MP and Ransom BR: New light on white matter. Stroke 34: 330-332, 2003

3. Pantoni L, Garcia JH and Gutierrez JA: Cerebral white matter is highly vulnerable to ischemia. Stroke 27: 1641-1647, 1996.
4. Back SA,Han BH, Luo NL, Chricton CA, Xanthoudakis S, Tam J, Arvin KL and Holtzman DM: Selective vulnerability of late oligodendrocyte progenitors to hypoxia-ischemia. J Neurosci 22: 455-463, 2002.

5. Dewar D, Underhill SM and Goldberg MP: Oligodendrocytes and ischemic brain injury. J Cereb Blood Flow Metab 23: 263-274, 2003.

6. Nishiyama A: Polydendrocytes: NG2 cells with many roles in development and repair of the CNS. Neuroscientist 13: 62-76, 2007.

7. Dawson MR, Polito A, Levine JM and Reynolds R: NG2-expressing glial progenitor cells: An abundant and widespread population of cycling cells in the adult rat CNS. Mol Cell Neurosci 24: 476-488, 2003.

8. Eugenín-von Bernhardi J and Dimou L: NG2-glia, more than progenitor cells. Adv Exp Med Biol 949: 27-45, 2016.

9. van Tilborg E, de Theije CGM, van Hal M, Wagenaar $\mathrm{N}$, de Vries LS, Benders MJ, Rowitch DH and Nijboer CH: Origin and dynamics of oligodendrocytes in the developing brain: Implications for perinatal white matter injury. Glia 66: 221-238, 2018.

10. Keirstead HS and Blakemore WF: The role of oligodendrocytes and oligodendrocyte progenitors in CNS remyelination. Adv Exp Med Biol 468: 183-197, 1999.

11. Nishiyama A, Komitova M, Suzuki R and Zhu X: Polydendrocytes (NG2 cells): Multifunctional cells with lineage plasticity. Nat Rev Neurosci 10: 9-22, 2009.

12. Tanaka K, Nogawa S, Suzuki S, Dembo T and Kosakai A: Upregulation of oligodendrocyte progenitor cells associated with restoration of mature oligodendrocytes and myelination in peri-infarct area in the rat brain. Brain Res 989: 172-179, 2003.

13. Franklin RJ: Why does remyelination fail in multiple sclerosis? Nat Rev Neurosci 3: 705-714, 2002.

14. Lubetzki C, Williams A and Stankoff B: Promoting repair in multiple sclerosis: Problems and prospects. Curr Opin Neurol 18: 237-244, 2005.

15. Ross SE, Greenberg ME and Stiles CD: Basic helix-loop-helix factors in cortical development. Neuron 39: 13-25, 2003.

16. Zhou Q, Wang S and Anderson DJ: Identification of a novel family of oligodendrocyte lineage-specific basic helix-loop-helix transcription factors. Neuron 25: 331-343, 2000.

17. Miller RH: Regulation of oligodendrocyte development in the vertebrate CNS. Prog Neurobiol 67: 451-467, 2002.

18. Jakovcevski I and Zecevic N: Olig transcription factors are expressed in oligodendrocyte and neuronal cells in human fetal CNS. J Neurosci 25: 10064-10073, 2005.

19. Zhou Q and Anderson DJ: The bHLH transcription factors OLIG2 and OLIG1 couple neuronal and glial subtype specification. Cell 109: 61-73, 2002.

20. Lu QR, Sun T, Zhu Z, Ma N, Garcia M, Stiles CD and Rowitch DH: Common developmental requirement for Olig function indicates a motor neuron/oligodendrocyte connection. Cell 109: 75-86, 2002.

21. Takebayashi H, Nabeshima Y, Yoshida S, Chisaka O, Ikenaka K and Nabeshima Y: The basic helix-loop-helix factor olig2 is essential for the development of motoneuron and oligodendrocyte lineages. Curr Biol 12: 1157-1163, 2002.

22. Arnett HA, Fancy SP, Alberta JA, Zhao C, Plant SR, Kaing S, Raine CS, Rowitch DH, Franklin RJ and Stiles CD: bHLH transcription factor Olig1 is required to repair demyelinated lesions in the CNS. Science 306: 2111-2115, 2004.

23. Dai J, Bercury KK, Jin W and Macklin WB: Olig1 Acetylation and nuclear export mediate oligodendrocyte development. J Neurosci 35: 15875-15893, 2015.

24. Niu J, Mei F, Wang L, Liu S, Tian Y, Mo W, Li H, Lu QR and Xiao L: Phosphorylated olig1 localizes to the cytosol of oligodendrocytes and promotes membrane expansion and maturation. Glia 60: 1427-1436, 2012

25. Tonchev AB, Yamashima T, Sawamoto $\mathrm{K}$ and Okano $\mathrm{H}$ : Transcription factor protein expression patterns by neural or neuronal progenitor cells of adult monkey subventricular zone. Neuroscience 139: 1355-1367, 2006.

26. Longa EZ, Weinstein PR, Carlson $S$ and Cummins R: Reversible middle cerebral artery occlusion without craniectomy in rats. Stroke 20: 84-91, 1989.

27. Bederson JB, Pitts LH, Tsuji M, Nishimura MC, Davis RL, Bartkowski H: Rat middle cerebral artery occlusion: Evaluation of the model and development of a neurologic examination. Stroke 17: 472-476, 1986.

28. Tu H, Deng L, Sun Q, Yao L, Han JS and Wan Y: Hyperpolarization-activated, cyclic nucleotide-gated cation channels: Roles in the differentialelectrophysiological properties of rat primary afferent neurons. J Neurosci Res 76: 713-722, 2004. 
29. Luo H, Cheng J, Han JS and Wan Y: Change of vanilloid receptor 1 expression in dorsal root ganglion and spinal dorsal horn during inflammatory nociception induced by complete Freund's adjuvant in rats. Neuroreport 15: 655-658, 2004

30. Jiang YQ, Xing GG, Wang SL, Tu HY, Chi YN, Li J, Liu FY, Han JS and Wan Y: Axonal accumulation of hyperpolarization-activated cyclic nucleotide-gated cation channels contributes to mechanical allodynia after peripheral nerve injury in rat Pain 137: 495-506, 2008

31. Song FE, Huang JL, Lin SH, Wang S, Ma GF and Tong XP: Roles of NG2-glia in ischemic stroke. CNS Neurosci Ther 23 . 547-553, 2017.

32. Mandai K, Matsumoto M, Kitagawa K, Matsushita K, Ohtsuki T, Mabuchi T, Colman DR, Kamada T and Yanagihara T: Ischemic damage and subsequent proliferation of oligodendrocytes in focal cerebral ischemia. Neuroscience 77: 849-861, 1997.

33. Chamberlain KA, Nanescu SE, Psachoulia K and Huang JK: Oligodendrocyte regeneration: Its significance in myelin replacement and neuroprotection in multiple sclerosis. Neuropharmacology 110: 633-643, 2016.

34. Bu J, Banki A, Wu Q and Nishiyama A: Increased NG2(+) glial cell proliferation and oligodendrocyte generation in the hypomyelinating mutant shiverer. Glia 4: 51-63, 2004.

35. Luessi F, Kuhlmann T and Zipp F: Remyelinating strategies in multiple sclerosis. Expert Rev Neurother 14: 1315-1334, 2014.

36. Paes de Faria J, Kessaris N, Andrew P, Richardson WD and Li H: New Olig1 null mice confirm a non-essential role for Olig1 in oligodendrocyte development. BMC Neurosci 15: 12, 2014

37. Qi Q, Zhang Y, Shen L, Wang R, Zhou J, Lü H and Hu J: Olig1 expression pattern in neural cells during rat spinal cord development. Neuropsychiatr Dis Treat 12: 909-916, 2016.

38. Sabo JK, Heine V, Silbereis JC, Schirmer L, Levison SW and Rowitch DH: Olig1 is required for noggin-induced neonatal myelin repair. Ann Neurol 81: 560-571, 2017.

39. Lu QR, Yuk D, Alberta JA, Zhu Z, Pawlitzky I, Chan J, McMahon AP, Stiles CD and Rowitch DH: Sonic hedgehogregulated oligodendrocyte lineage genes encoding bHLH proteins in the mammalian central nervous system. Neuron 25 317-329, 2000

40. Ligon KL, Kesari S, Kitada M, Sun T, Arnett HA, Alberta JA, Anderson DJ, Stiles CD and Rowitch DH: Development of NG2 neural progenitor cells requires Olig gene function. Proc Nat Acad Sci USA 103: 7853-7858, 2006.

41. Dawson MR, Levine JM and Reynolds R: NG2-expressing cells in the central nervous system: Are they oligodendroglial progenitors? J Neurosci Res 61: 471-479, 2000.

42. Peters $A$ and Sethares C: Is there remyelination during aging of the primate central nervous system? J Comp Neurol 460 : 238-254, 2003

43. Franklin RJ, Zhao C and Sim FJ: Ageing and CNS remyelination. Neuroreport 13: 923-928, 2002.
44. Cheng T, Xue X and Fu J: Effect of Olig1 on the development of oligodendrocytes and myelination in a neonatal rat PVL model induced by hypoxia-ischemia. Mol Med Rep 11: 2379-2386, 2015.

45. Zhao H, Gao XY, Wang DX and Zhang YB: Effect of adenovirus-mediated gene transfer of Olig1 on oligodendrocyte differentiation and remyelination in a rat model of focal cerebral ischemia. Neural Regen Res 4: 862-867, 2009.

46. He L and Lu QR: Coordinated control of oligodendrocyte development by extrinsic and intrinsic signaling cues. Neurosci Bull 29: 129-143, 2013.

47. Fancy SP, Zhao C and Franklin RJ: Increased expression of Nkx2.2 and Olig2 identifies reactive oligodendrocyte progenitor cells responding to demyelination in the adult CNS. Mol Cell Neurosci 27: 247-254, 2004.

48. Balasubramaniyan V, Timmer N, Kust B, Boddeke E and Copray S: Transient expression of Olig1 initiates the differentiation of neural stem cells into oligodendrocyte progenitor cells. Stem Cells 22: 878-882, 2004.

49. Watanabe M, Hadzic T and Nishiyama A: Transient upregulation of $\mathrm{Nkx} 2.2$ expression in oligodendrocyte lineage cells during remyelination. Glia 46: 311-322, 2004.

50. Soundarapandian MM, Selvaraj V, Lo UG, Golub MS, Feldman DH, Pleasure DE and Deng W: Zfp488 promotes oligodendrocyte differentiation of neural progenitor cells in adult mice after demyelination. Sci Rep 1: 2, 2011.

51. Finzsch M, Stolt CC, Lommes P and Wegner M: Sox9 and Sox 10 influence survival and migration of oligodendrocyte precursors in the spinal cord by regulating PDGF receptor alpha expression. Development 135: 637-646, 2008.

52. Talbott JF, Loy DN, Liu Y, Qiu MS, Bunge MB, Rao MS and Whittemore SR: Endogenous Nkx $2.2^{+} / \mathrm{Olig} 2^{+}$oligodendrocyte precursor cells fail to remyelinate the demyelinated adult rat spinal cord in the absence of astrocytes. Exp Neurol 192: 11-24, 2005.

53. Carmen J, Magnus T, Cassiani-Ingoni R, Sherman L, Rao MS and Mattson MP: Revisiting the astrocyte-oligodendrocyte relationship in the adult CNS. Prog Neurobiol 82: 151-162, 2007.

54. Haindl MT, Köck U, Zeitelhofer-Adzemovic M, Fazekas F and Hochmeister S: The formation of a glial scar does not prohibit remyelination in an animal model of multiple sclerosis. Glia 67: 467-481, 2019.

55. Williams A, Piaton G and Lubetzki C: Astrocytes-friends or foes in multiple sclerosis? Glia 55: 1300-1312, 2007.

56. Kotter MR, Li WW, Zhao C and Franklin RJ: Myelin impairs CNS remyelination by inhibiting oligodendrocyte precursor cell differentiation. J Neurosci 26: 328-332, 2006.

This work is licensed under a Creative Commons Attribution-NonCommercial-NoDerivatives 4.0 International (CC BY-NC-ND 4.0) License. 